DOI: $10.15593 / 24111678 / 2016.02 .02$

УДК 623.437.3.093; 629.1.032.001; 629.36

\author{
Р.Ю. Добрецов ${ }^{1}$, Е.Г. Борисов ${ }^{2}$, С.И. Матросов ${ }^{2}$ \\ ${ }^{1}$ Санкт-Петербургский государственный политехнический \\ университет Петра Великого, Санкт-Петербург, Россия \\ ${ }^{2}$ Научно-техническое ЗАО «Ровер», Санкт-Петербург, Россия \\ О ВЫБОРЕ ТИПА ПРИВОДА
ДЛЯ СФЕРИЧЕСКОГО РОБОТА
}

\begin{abstract}
Рассмотрен вопрос обоснования выбора технического решения при проектировании шасси сфрерического робота, реализующего наибольшую точность отработки предписанной траектории движения. Робот предназначен для работы в составе группы сферических роботов, объединенной под контролем мультиагентной системы управления. Гетерогенная группа может объединять машины наземного базирования, а также содержать воздушную компоненту. Назначение группы - ведение экологического мониторинга или патрулирование территорий предприятий и других объектов, как внутри помещений, так и на подготовленных площадках. Рассмотрены известные технические решения по организации привода сферических роботов. На основании анализа информационных источников предложена номенклатура частных показателей, являющихся основой для качественного и количественного сравнения вариантов конструкции шасси. Выставлены балльные (ранговые) оценки для сравниваемых вариантов. Предложен алгоритм формирования оценочного показателя на основе метода экспертных оценок. С использованием данного алгоритма произведена сравнительная оценка отобранных вариантов конструкции и сделан выбор наиболее перспективного технического решения.
\end{abstract}

Ключевые слова: сферический робот, транспортное средство, движитель, подвижность, устойчивость, управляемость.

\author{
R.lu. Dobretsov ${ }^{1}$, E.G. Borisov ${ }^{2}$, S.I. Matrosov ${ }^{2}$ \\ ${ }^{1}$ Peter the Great St.Petersburg Polytechnic University, \\ St.Petersburg, Russian Federation \\ ${ }^{2}$ Science and Technology Rover Company Ltd, St.Petersburg, Russian Federation

\section{ON THE CHOICE OF THE TYPE OF ACTUATOR FOR THE SPHERICAL ROBOT}

Consider the rationale for the choice of technical solutions for designing the chassis of a spherical robot that implements the highest accuracy of the prescribed trajectory. The robot is designed to work as part of a group of spherical robots United under the control of multi-agent control system. A heterogeneous group can combine land-based machines and to support the air component. Group assignment - maintenance of environmental monitoring or patrolling of the territories of the enterprises and other objects inside or on prepared sites. Describes the known technical solutions for the organization of driven spherical robots. Based on the analysis of information sources, the proposed range of partial indicators which are the basis for qualitative and quantitative comparison of variants chassis design. Exhibited scoring (ranked) scores for the compared variants. The algorithm of formation of evaluation index on the basis of method of expert estimations. Using this algorithm comparative evaluation of the selected options of design and made a selection of the most promising technical solutions.

Keywords: spherical robot, vehicle, propulsion, mobility, stability, controllability. 
Цель данной работы - предложить методику количественной оценки рассматриваемых технических решений при разработке концепции компоновки сферического робота.

Показано, что шасси сферического робота является перспективным для использования под размещение оборудования для ведения мониторинга экологической обстановки на производстве при нормальном протекании технологических процессов и при возникновении нештатных ситуаций, а также для ведения наблюдения при патрулировании различных объектов $[1,2]$. Шасси предназначено преимущественно для работы в помещениях или на подготовленных горизонтальных площадках (автомобильные стоянки, открытые дворы и др.).

Отличительными особенностями шасси сферического робота являются высокая защищенность бортового оборудования от агрессивного воздействия внешней среды, высокая экономичность при удовлетворительной подвижности. Значимые недостатки - малая грузоподъемность и проблема точности отработки заданной траектории.

Первый недостаток можно нивелировать, используя сферический робот в составе группы, в идеале объединенной мультиагентной системой управления [3]: роботы, составляющие гетерогенную группу, могут нести различное оборудование, которое согласованно используется для изучения объекта интереса. В настоящее время показано, что работа групп роботов может быть эффективной, проанализированы стратегии и сформулированы задачи группового управления [4]. Решение этих задач применительно к группам сферических роботов - проблема, выходящая за рамки данной статьи.

Второй недостаток может быть преодолен за счет выбора типа привода шасси. Для поиска привода, позволяющего реализовать наиболее точное управление траекторией сферического робота, на основе анализа литературных источников [5-15] и результатов опытноконструкторских разработок ЗАО «Ровер» в области создания сферических роботов проведена типизация технических решений в области их конструирования и сформулирован список основных параметров сравнения конструкций (рисунок).

При первичном сравнении вариантов достаточно проведения оценки на основе опроса группы независимых экспертов. В качестве экспертов выступают основные участники проекта. 


\begin{tabular}{|c|c|c|c|c|c|c|c|c|c|c|c|}
\hline \multicolumn{12}{|c|}{$\begin{array}{c}\text { Частные показатели сравнительной оценки конструктивных решений } \\
\text { для шасси сферического робота }\end{array}$} \\
\hline \multicolumn{3}{|c|}{ Проходимость } & \multicolumn{3}{|c|}{ Управляемость } & \multicolumn{3}{|c|}{$\begin{array}{c}\text { Общетехнические } \\
\text { показатели }\end{array}$} & \multicolumn{3}{|c|}{$\begin{array}{c}\text { Экономические } \\
\text { показатели }\end{array}$} \\
\hline 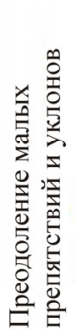 & 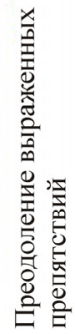 & 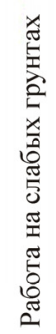 & 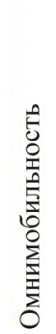 & 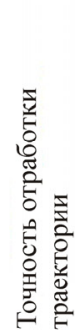 & 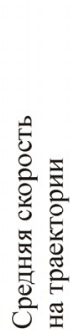 & 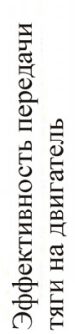 & 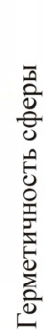 & 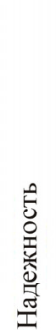 & 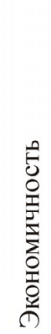 & $\overbrace{0}^{0}$ & $\begin{array}{l}\hat{E} \\
0 \\
0 \\
\delta \\
\delta \\
b\end{array}$ \\
\hline
\end{tabular}

Рис. Система параметров сравнительной оценки вариантов компоновки сферических роботов

Используемая шкала оценок:

5 - свойство обеспечено в максимальной степени (отлично);

4 - промежуточная оценка (хорошо);

3 - свойство обеспечено в минимальной степени (удовлетворительно).

Ниже приведена номенклатура опробованных и перспективных технических решений по исполнению приводов сферических роботов. Присваиваем каждому типу привода буквенный код:

A. Роликовый [5] (контактный привод от роликового механизма или колес омни-тележки).

$B$. Маятниковый двухплоскостной [5-10] (бесконтактный привод, движение происходит за счет изменения положения центра тяжести робота, вызываемого отклонением маятника, имеющего две степени свободы).

$C$. Маятниковый трехплоскостной $[10,11]$ (от предыдущего варианта отличается наличием трех степеней свободы у маятника).

$D$. Гироскопический $[5,12,13]$ (перемещение вызывается опрокидывающим моментом, возникающим при совместной работе двух или трех гироскопов).

$E$. Аэродинамический (сила тяги создается пропеллером).

$F$. Деформация сферы или «псевдосферы» $[5,14]$ (качение возникает как следствие управляемого формоизменения оболочки). 
$G$. Шагающий [15] (движитель-трансформер, в режиме качения движение происходит за счет момента, создаваемого гироскопами, в режиме шагания - передвижение по принципу шагающего механизма).

Рассмотрим номенклатуру параметров для сравнительной оценки (см. рисунок, табл. 1).

1. Эффективность преодоления малых препятствий. Малые препятствия и уклоны - препятствия высотой/глубиной до 5-10 \% от радиуса сферы, уклоны до 5-10.

2. Эффективность преодоления выраженных препятствий. Выраженные препятствия - ступени, бордюры и другие одиночные препятствия, по размерам соизмеримые с радиусом сферы.

Т а блиц ц 1

Весовые коэффициенты параметров сравнительной оценки и их групп

\begin{tabular}{|l|c|c|c|c|c|c|c|c|c|c|}
\hline \multirow{2}{*}{ Группа параметров, параметры } & \multicolumn{7}{|c|}{ Код } \\
\cline { 2 - 9 } & $A$ & $B$ & $C$ & $D$ & $E$ & $F$ & $G$ & $k_{1}$ & $k_{2}$ & $k_{1} \times k_{2}$ \\
\hline Проходимость: & & & & & & & & 0,25 & & \\
\hline $\begin{array}{l}\text { - преодоление малых препятст- } \\
\text { вий и уклонов }\end{array}$ & 4 & 3 & 4 & 4 & 5 & 3 & 5 & & 0,50 & 0,1250 \\
\hline $\begin{array}{l}\text { - преодоление выраженных пре- } \\
\text { пятствий }\end{array}$ & 3 & 3 & 3 & 5 & 5 & 3 & 5 & & 0,20 & 0,0500 \\
\hline - работа на слабых грунтах & 4 & 5 & 5 & 5 & 3 & 4 & 4 & & 0,30 & 0,0750 \\
\hline Управляемость: & & & & & & & & 0,25 & & \\
\hline - омнимобильность & 5 & 4 & 5 & 4 & 5 & 5 & 4 & & 0,40 & 0,1000 \\
\hline $\begin{array}{l}\text { - точность отработки траекто- } \\
\text { рии }\end{array}$ & 5 & 4 & 5 & 4 & 3 & 4 & 4 & & 0,40 & 0,1000 \\
\hline $\begin{array}{l}\text { - средняя скорость на траекто- } \\
\text { рии }\end{array}$ & 4 & 4 & 5 & 5 & 5 & 3 & 4 & & 0,20 & 0,0500 \\
\hline Общетехнические показатели: & & & & & & & & 0,25 & & \\
\hline $\begin{array}{l}\text { - эффективность передачи тяги } \\
\text { на движитель }\end{array}$ & 5 & 4 & 4 & 4 & 5 & 4 & 5 & & 0,33 & 0,0825 \\
\hline - герметичность сферы & 5 & 5 & 5 & 5 & 3 & 4 & 3 & & 0,33 & 0,0825 \\
\hline - надежность & 5 & 5 & 5 & 4 & 3 & 3 & 3 & & 0,33 & 0,0825 \\
\hline Экономические показатели: & & & & & & & & 0,25 & & \\
\hline - экономичность & 5 & 5 & 5 & 3 & 3 & 4 & 4 & & 0,50 & 0,1250 \\
\hline - ресурс & 5 & 5 & 5 & 4 & 3 & 3 & 3 & & 0,30 & 0,0750 \\
\hline - стоимость & 5 & 5 & 4 & 3 & 3 & 3 & & 0,20 & 0,0500 \\
\hline
\end{tabular}


3. Эффективность работы на слабых грунтах. Слабые грунты грунты со слабым поверхностным слоем, характеризующиеся низкой несущей способностью и высоким сопротивлением движению (песок, снег, пересыщенный влагой деструктурированный торф и др.).

4. Омнимобильность - способность оперативно изменять траекторию движения (фактически быть готовым в любой момент изменить направление движение заданным образом).

5. Точность отработки траектории - способность сферического робота воспроизвести заданную оператором (системой управления) траекторию с минимальным отклонением и реагировать на изменение направления вектора тяги.

6. Средняя скорость на траектории - средняя скорость на заданном маршруте.

7. Эффективность передачи силы тяги - возможность реализовать тяговое усилие по условиям передачи крутящего момента на сферу. Например, для роликового привода передаваемый момент (следовательно, сила тяги) ограничен силой прижатия ролика к сфере, массой стабилизированной платформы и др.; для маятникового привода - массой и размахом маятника.

8. Возможность обеспечения герметичности сферы.

9. Надежность - включает в себя собственно надежность механизмов сферического робота и уязвимость по отношению к влиянию внешних факторов (например, попадание влаги и посторонних предметов внутрь сферы).

10. Экономичность привода - может быть оценена на основе сравнения пробега роботов с разными типами привода в одинаковых условиях эксплуатации.

11. Ресурс - в первом приближении может оцениваться на основе сведений о гарантийном сроке эксплуатации.

12. Стоимость - определяется оценочной себестоимостью изделия.

Малочисленность экспертной группы не дает возможности корректно использовать математический аппарат метода экспертных оценок $[16,17]$, поэтому при формировании итоговой оценки, выставленной группой экспертов, применяется следующий метод обработки частных оценок, построенный по аналогии с одним из методов, применяемых для оценки эффективности военно-транспортных машин. Альтернативой данного метода является метод Харрингтона [17], также применяемый при сравнении конструкций шасси транспортных машин 
(например, с позиции энергоэффективности [18]). Выбор сделан в пользу метода экспертных оценок ввиду наличия группы экспертов и сравнительно небольшого перечня частных показателей. Однако метод Харрингтона окажется предпочтительным в случае необходимости перехода к количественному сравнению рассматриваемых вариантов технических решений.

Построение показателя сравнительной оценки формализовано в виде следующего алгоритма:

1. Рассматриваемые параметры разбиваются на четыре базовые группы (см. табл. 1). Каждой группе присваивается весовой коэффициент, исходя из оценки экспертами ее значимости в контексте решаемой задачи. Сумма групповых коэффициентов равна единице:

$$
\sum_{i=1}^{N_{1}}\left(k_{1}\right)_{i}=1, \quad N_{1}=4
$$

2. Каждому рассматриваемому параметру присваивается весовой коэффициент (см. табл. 1), исходя из оценки экспертами его значимости в контексте решаемой задачи. Сумма коэффициентов внутри группы равна единице:

$$
\sum_{j=1}^{N_{2}}\left(k_{2}\right)_{j}=1, \quad N_{2}=3
$$

3. Для каждого параметра вычисляется произведение $\left(k_{1}\right)_{i} \cdot\left(k_{2}\right)_{j}$ (см. табл. 1).

4. Промежуточная оценка выставляется по результатам попарного сравнения вариантов (табл. 2). Индекс $l=\overline{1,7}$ присваивается сравниваемым вариантам $A, B, C, D, E, F, G$.

Таблица 2

Кодировка вариантов попарных сравнений

\begin{tabular}{|c|c|c|c|c|c|c|c|}
\hline & $A$ & $B$ & $C$ & $D$ & $E$ & $F$ & $G$ \\
\hline$A$ & & $A B$ & $A C$ & $A D$ & $A E$ & $A F$ & $A G$ \\
\hline$B$ & & & $B C$ & $B D$ & $B E$ & $B F$ & $G F$ \\
\hline$C$ & & & & $C D$ & $C E$ & $C F$ & $C G$ \\
\hline$D$ & & & & & $D E$ & $D F$ & $D G$ \\
\hline$E$ & & & & & & $E F$ & $E G$ \\
\hline$F$ & & & & & & & $F G$ \\
\hline
\end{tabular}


Пример сравнения приведен в табл. 3. Если один из вариантов имеет преимущество с точки зрения экспертов, ему отдают балл $t_{l m}=\left(k_{1}\right)_{i} \cdot\left(k_{2}\right)_{j}, \quad m=\overline{1, i \cdot j}$ целиком (проигравшему варианту остается $\left.t_{l m}=0\right)$. Если экспертные оценки вариантов одинаковые, балл делится между конкурентами пополам: $t_{l m}=0,5 \cdot\left(k_{1}\right)_{i} \cdot\left(k_{2}\right)_{j}$.

Таблица 3

Пример попарных сравнений: пара $A C$

\begin{tabular}{|l|c|c|c|c|c|c|c|}
\hline \multirow{2}{*}{$\begin{array}{c}\text { Группа параметров, } \\
\text { паретры }\end{array}$} & \multicolumn{7}{|c|}{ Код } \\
\cline { 2 - 8 } & $A$ & $C$ & $k_{1}$ & $k_{2}$ & $k_{1} \times k_{2}$ & $A$ & $C$ \\
\hline Проходимость: & & & 0,25 & & & & \\
\hline $\begin{array}{l}\text { - преодоление малых препятст- } \\
\text { вий и уклонов }\end{array}$ & 4 & 4 & & 0,50 & 0,1250 & 0,0625 & 0,0625 \\
\hline $\begin{array}{l}\text { - преодоление выраженных пре- } \\
\text { пятствий }\end{array}$ & 3 & 3 & & 0,20 & 0,0500 & 0,0250 & 0,0250 \\
\hline Работа на слабых грунтах & 4 & 5 & & 0,30 & 0,0750 & 0 & 0,0750 \\
\hline Управляемость: & & & 0,25 & & & & \\
\hline - омнимобильность & 5 & 5 & & 0,40 & 0,1000 & 0,0500 & 0,0500 \\
\hline - точность отработки траектории & 5 & 5 & & 0,40 & 0,1000 & 0,0500 & 0,0500 \\
\hline - средняя скорость на траектории & 4 & 5 & & 0,20 & 0,0500 & 0 & 0,0500 \\
\hline Общетехнические показатели: & & & 0,25 & & & & \\
\hline $\begin{array}{l}\text { - эффективность передачи тяги } \\
\text { на движитель }\end{array}$ & 5 & 4 & & 0,33 & 0,0825 & 0,0825 & 0 \\
\hline - герметичность сферы & 5 & 5 & & 0,33 & 0,0825 & 0,0413 & 0,0413 \\
\hline - надежность & 5 & 5 & & 0,33 & 0,0825 & 0,0413 & 0,0413 \\
\hline - экономические показатели: & & & 0,25 & & & & \\
\hline - экономичность & 5 & 5 & & 0,50 & 0,1250 & 0,0625 & 0,0625 \\
\hline - ресурс & 5 & 5 & & 0,30 & 0,0750 & 0,0375 & 0,0375 \\
\hline - стоимость & 5 & 5 & & 0,20 & 0,0500 & 0,0250 & 0,0250 \\
\hline - суммарный балл & & & & & 0,4775 & 0,5200 \\
\hline
\end{tabular}

5. Производится суммирование набранных в ходе попарного сравнения баллов для каждого варианта:

$$
T_{l}=\sum_{m=1}^{N_{3}} t_{l m}, \quad N_{3}=i \cdot j=12 .
$$


Выигравшим считается тот вариант, значение $T_{l}$ для которого оказалось больше. В табл. 4 приведены коды «победителей» попарного сравнения в соответствии с табл. 2.

Таблица 4

«Победители» попарных сравнений

\begin{tabular}{|c|c|c|c|c|c|c|c|}
\hline & $A$ & $B$ & $C$ & $D$ & $E$ & $F$ & $G$ \\
\hline$A$ & & $A$ & $C$ & $A$ & $A$ & $A$ & $A$ \\
\hline$B$ & & & $C$ & $B$ & $B$ & $B$ & $G$ \\
\hline$C$ & & & & $C$ & $C$ & $C$ & $C$ \\
\hline$D$ & & & & & $D$ & $D$ & $D$ \\
\hline$E$ & & & & & & $F$ & $G$ \\
\hline$F$ & & & & & & & $G$ \\
\hline
\end{tabular}

6. По табл. 4 производится распределение условных позиций рейтинга. В случае одинаковой частоты «выигрыша» для двух конкурентов предпочтение отдается тому варианту, который побеждает в попарном сравнении между конкурентами (табл. 5). Аналогично поступают при наличии большего числа конкурентов. В табл. 5 с увеличением номера позиции рейтинга растет предпочтительность варианта.

Таблица 5

Итоговый рейтинг

\begin{tabular}{|c|c|c|c|c|c|c|}
\hline$A$ & $B$ & $C$ & $D$ & $E$ & $F$ & $G$ \\
\hline 6 & 5 & 7 & 4 & 1 & 2 & 3 \\
\hline
\end{tabular}

Таким образом, на основании сравнения вариантов сделан вывод: для решения задач мониторинга (и подобных ей) внутри подготовленных помещений или в условиях искусственной среды (городская улица, двор, обработанное поле) оптимальным представляется использование маятникового привода с возможностью позиционирования маятника по трем независимым координатам (вариант под кодом $C$ ).

Таким образом, проведенные проектно-конструкторские работы и выполненные расчеты позволяют сделать следующие основные выводы: 
1. Разработанная номенклатура частных показателей для сравнения конструкций приводов сферических роботов может быть применена на практике.

2. При построении обобщенного оценочного показателя целесообразно применение метода экспертной оценки или метода Харрингтона.

3. При проектировании сферического робота, реализующего высокую точность отработки траектории движения, предпочтительным является использование маятникового привода, имеющего три степени свободы.

Работа выполнена при финансовой поддержке Минобрнауки России в рамках Федеральной иелевой программы «Исследования и разработки по приоритетным направлениям развития научнотехнологического комплекса России на 2014-2020 годыр» по проекту: «Разработка методов и алгоритмов адаптивного управления движением мультиагентных сферических роботов повыменной маневренности в условиях неопределенности и существенных внешних возмущений» (уникальный идентификатор проекта RFMEFI61315X0047).

The work is executed at financical support of the Ministry of education and science under the Federal target program "Research and development on priority directions of development of scientific-technological complex of Russia for 2014-2020"project: "Development of methods and algorithms of adaptive multi-agent motion control of spherical robots enhanced agility in the face of uncertainty and significant external disturbances" (unique identifier of project RFMEFI61315X0047).

\section{Список литературы}

1. Сферический робот как платформа для ведения экологического мониторинга / Р.Ю. Добрецов, Е.Г. Борисов [и др.] // Транспорт. Транспортные сооружения. Экология. - 2015. - № 3. - С. 35-50.

2. Добрецов Р.Ю., Васильев И.В. Силовой и мощностной баланс сферического движителя // Неделя науки СПбПУ: материалы форума с международным участием / Ин-т энергетики и транспортных систем. - СПб.: Изд-во Политехн. ун-та, 2015. - Ч. 1. - С. 30-33.

3. Борисов Е.Г., Добрецов Р.Ю., Матросов С.И. О наземной мультиагентной системе на базе группы сферических роботов // Транспорт- 
ные и транспортно-технологические системы: материалы Междунар. науч.-техн. конф. / ТюмГНГУ. - Тюмень, 2016. - С. 32-37.

4. Каляев И.А., Гайдук А.Р., Капустян С.Г. Модели и алгоритмы коллективного управления в группах роботов. - М.: Физматлит, 2009. $278 \mathrm{c}$.

5. Chase R., Pandya A. A Review of Active Mechanical Driving Principles of Spherical Robots // Robotics. - 2012. - No. 1. - P. 3-23. DOI:10.3390/robotics 1010003

6. Робот-шар ROSPHERE [Электронный ресурс]. - URL: http:// www.prorobot.ru/09/ROSPHERE_shar.php (дата обращения: 17.05.2016).

7. Rotundus. GroundBot ${ }^{\mathrm{TM}}$. - URL: http://www.rotundus.se/ (дата обращения: 17.05.2016).

8. Modeling and Simulation of a Spherical Mobile Robot / Sang Shengju, Zhao Jichao, Wu Hao, Chen Shoujun, An Qi // ComSIS. - February 2010. - Vol. 7, no. 1, Special Issue. - P. 51-62.

9. Kinetic Model for a Spherical Rolling Robot with Soft Shell in a Beeline Motion / Zhang Sheng, Fang Xiang, Zhou Shouqiang, Du Kai // Journal of Multimedia. - February 2014. - Vol. 9, no. 2. - P. 223-229. DOI: 10.4304/jmm.9.2.223-229

10. Chunfeng Yue, Shuxiang Guo, Liwei Shi. Hydrodynamic Analysis of the Spherical Underwater Robot SUR-II // International Journal of Advanced Robotic Systems. - 2013. - Vol. 10, no. 247. - P. 1-12. DOI: $10.5772 / 56524$

11. Moving Eye - interactive Telepresence over internet with a Ball Shaped mobile Robot Publication type / Panu Harmo, Aarne Halme, Hannu Pitkänen, Petri Virekoski, Matias Halinen, Jussi Suomela // IFAC Telematic Conference. - 2001. - P. 1-6.

12. Unusual off-road locomotion. - URL: http://www. unusuallocomotion.com/medias/images/thisthe-sphere-robot-1993.jpg?fx=r_ 900_900 (дата обращения: 17.05.2016).

13. Gigantic Robotic Ball. - URL: https://www.flickr.com/photos/ daikiki/3596965040/ (дата обращения: 17.05.2016).

14. National Robotics Engineering Center. - URL: http://www.nrec.ri. cmu.edu/projects/rats/ (дата обращения: 17.05.2016).

15. MorphHex MK-II. - URL: http://blog. trossenrobotics.com/2014/ 03/19/morphex-mk-ii-in-action/ (дата обращения: 17.05.2016).

16. Орлов А.И. Теория принятия решений: учеб. пособие. - М.: Март, 2004. - 656 c. 
17. Адлер Ю.П., Маркова Е.В., Грановский Ю.В. Планирование эксперимента при поиске оптимальных условий. - М.: Наука, 1976. $280 \mathrm{c}$.

18. Добрецов Р.Ю. Объективная оценка технических характеристик шасси транспортных гусеничных машин // Тракторы и сельскохозяйственные машины. - 2011. - № 2. - С. 19-23.

\section{References}

1. Dobretsov R.Iu., Borisov E.G. [et al.]. Sfericheskii robot kak platforma dlia vedeniia ekologicheskogo monitoringa [A spherical robot as a platform for conducting environmental monitoring]. Transport. Transportnye sooruzheniia. Ekologiia, 2015, no. 3, pp. 35-50.

2. Dobretsov R.Iu., Vasil'ev I.V. Silovoi i moshchnostnoi balans sfericheskogo dvizhitelia [Power and power balance of spherical robot]. $M a$ terialy foruma s mezhdunarodnym uchastiem "Nedelia nauki SPbPU". Saint Petersburg: Politekhnicheskii universitet, 2015, vol. 1. 352 p.

3. Borisov E.G., Dobretsov R.Iu., Matrosov S.I. O nazemnoi mul'tiagentnoi sisteme na baze gruppy sfericheskikh robotov [On ground-based multi-agent system on the basis of the group of spherical robots]. Materialy Mezhdunarodnoi nauchno-tekhnicheskoi konferentsii "Transportnye $i$ transportno-tekhnologicheskie sistemy". Tiumenskii gosudarstvennyi neftegazovyi universitet, 2016. 329 p.

4. Kaliaev I.A., Gaiduk A.R., Kapustian S.G. Modeli i algoritmy kollektivnogo upravleniia $\mathrm{v}$ gruppakh robotov [Models and algorithms of collective control in groups of robots]. Moscow: Fizmatlit, 2009. 278 p.

5. Chase R., Pandya A. A Review of Active Mechanical Driving Principles of Spherical Robots. Robotics, 2012, no. 1, pp. 3-23. DOI:10.3390/robotics1010003

6. Robot-shar ROSPHERE [Robot-sphere ROSPHERE], available at: http://www.prorobot.ru/09/ROSPHERE_shar.php (accessed 17 May 2016).

7. Rotundus. GroundBot ${ }^{\mathrm{TM}}$, available at: http://www.rotundus.se/ (accessed 17 May 2016).

8. Sang Shengju, Zhao Jichao, Wu Hao, Chen Shoujun, An Qi. Modeling and Simulation of a Spherical Mobile Robot. ComSIS, 2010, vol. 7, no. 1, Special Issue, pp. 51-62.

9. Zhang Sheng, Fang Xiang, Zhou Shouqiang, Du Kai. Kinetic Model for a Spherical Rolling Robot with Soft Shell in a Beeline Motion. 
Journal of Multimedia, February 2014, vol. 9, no. 2, pp. 223-229. DOI: 10.4304/jmm.9.2.223-229

10. Chunfeng Yue, Shuxiang Guo, Liwei Shi. Hydrodynamic Analysis of the Spherical Underwater Robot SUR-II. International Journal of Advanced Robotic Systems, 2013, vol. 10, no. 247, pp. 1-12. DOI: $10.5772 / 56524$

11. Panu Harmo, Aarne Halme, Hannu Pitkänen, Petri Virekoski, Matias Halinen, Jussi Suomela Moving Eye - interactive Telepresence over internet with a Ball Shaped mobile Robot Publication type. IFAC Telematic Conference, 2001, pp. 1-6.

12. Unusual off-road locomotion, available at: http://www. unusuallocomotion.com/medias/images/thisthe-sphere-robot-1993.jpg? $\mathrm{fx}=\mathrm{r}_{-}$ 900_900 (accessed 17 May 2016).

13. Gigantic Robotic Ball, available at: https://www.flickr.com/ photos/daikiki/3596965040/ (accessed: 17 May 2016).

14. National Robotics Engineering Center, available at: http://www.nrec.ri.cmu.edu/projects/rats/ (accessed 17 May 2016).

15. MorphHex MK-II, available at: http://blog.trossenrobotics.com/ 2014/03/19/ morphex-mk-ii-in-action/ (accessed 17 May 2016).

16. Orlov A.I. Teoriia priniatiia reshenii [The theory of decisionmaking]. Moscow: Mart, 2004. 656 p.

17. Adler Iu.P., Markova E.V., Granovskii Iu.V. Planirovanie eksperimenta pri poiske optimal'nykh uslovii [Planning of experiment when searching optimal conditions]. Moscow: Nauka, 1976. 280 p.

18. Dobretsov R.Iu. Ob"ektivnaia otsenka tekhnicheskikh kharakteristik shassi transportnykh gusenichnykh mashin [Objective assessment of the technical characteristics of the chassis of the tracked vehicle]. Traktory $i$ sel'skokhoziaistvennye mashiny, 2011, no. 2, pp. 19-23.

Получено 25.05.2016

\section{Об авторах}

Добрецов Роман Юрьевич (Санкт-Петербург, Россия) - кандидат технических наук, доцент, профессор кафедры «Инжиниринг силовых установок и транспортных средств» Санкт-Петербургского государственного политехнического университета Петра Великого (195251, Санкт-Петербург, ул. Политехническая, 29, e-mail: dr-idpo@yandex.ru). 
Борисов Евгений Геннадьевич (Санкт-Петербург, Россия) кандидат технических наук, доцент, ведущий научный сотрудник Санкт-Петербургского государственного университета телекоммуникаций им. проф. М.А. Бонч-Бруевича, главный научный сотрудник ЗАО «Ровер» (198323, Санкт-Петербург, ул. Заречная (Горелово), 2, e-mail: begspb1967@mail.ru).

Матросов Сергей Ильич (Санкт-Петербург, Россия) - почетный машиностроитель РФ, генеральный директор ЗАО «Ровер» (198323, Санкт-Петербург, ул. Заречная (Горелово), 2, e-mail: rover@ peterlink.ru).

\section{About the authors}

Roman Iu. Dobretsov (St.Petersburg, Russian Federation) - Ph.D. in Technical Sciences, Associate Professor, Department of Engineering of Engines and Transport Vehicles, Peter the Great St.Petersburg Polytechnic University (29, Politechnicheskaia st., St.Petersburg, 195251, Russian Federation, e-mail: dr-idpo@yandex.ru).

Evgenii G. Borisov (St.Petersburg, Russian Federation) - Ph.D. in Technical Sciences, Associate Professor, Leading Researcher, St.Petersburg Telecommunication University, Rover Company Ltd (2, Zaretshnaia st., St.Petersburg, Russian Federation, 198323, e-mail: begspb1967@ mail.ru).

Sergei I. Matrosov (St.Petersburg, Russian Federation) - Honorable Mashine Engineer of Russian Federation, Director General, Rover Company Ltd (2, Zarechnaia str., St.Petersburg, 198323, Russian Federation, e-mail: rover@peterlink.ru). 